\title{
3-D Directional Transverse Oscillations Synthetic Aperture Vector Flow Imaging with a 1024 Element Matrix Probe
}

Stuart, Matthias Bo; Jensen, Jørgen Arendt

Published in:

Proceedings of 2019 IEEE International Ultrasonics Symposium

Link to article, DOI:

10.1109/ULTSYM.2019.8926133

Publication date:

2019

Document Version

Peer reviewed version

Link back to DTU Orbit

Citation (APA):

Stuart, M. B., \& Jensen, J. A. (2019). 3-D Directional Transverse Oscillations Synthetic Aperture Vector Flow Imaging with a 1024 Element Matrix Probe. In Proceedings of 2019 IEEE International Ultrasonics Symposium (pp. 364-367). IEEE. https://doi.org/10.1109/ULTSYM.2019.8926133

\section{General rights}

Copyright and moral rights for the publications made accessible in the public portal are retained by the authors and/or other copyright owners and it is a condition of accessing publications that users recognise and abide by the legal requirements associated with these rights.

- Users may download and print one copy of any publication from the public portal for the purpose of private study or research.

- You may not further distribute the material or use it for any profit-making activity or commercial gain

- You may freely distribute the URL identifying the publication in the public portal 


\title{
3-D Directional Transverse Oscillations Synthetic Aperture Vector Flow Imaging with a 1024 Element Matrix Probe
}

\author{
Matthias Bo Stuart and Jørgen Arendt Jensen \\ Center for Fast Ultrasound Imaging, DTU Health Technology, Technical University of Denmark
}

\begin{abstract}
D vector flow imaging is simulated with a 1024 element matrix array and vessels aligned with the $x$ - and $y$ axes. Parabolic flow profiles with a peak velocity of $0.5 \mathrm{~m} / \mathrm{s}$ are simulated with an interleaved synthetic aperture sequence with 5 emissions at a pulse repetition frequency of $15 \mathrm{kHz}$. Two-peak receive apodizations are used to induce transverse oscillations (TO) in the $x$ - and $y$-directions, and a directional TO velocity estimator based on cross-correlation is used to estimate the 3 velocity components. The relative mean bias and relative mean standard deviation are calculated for each velocity component and the velocity magnitude. For the vessel aligned with the $x$-axis, the bias of the $x$-component is $\mathbf{- 1 0 . 1 \%}$, and for the vessel aligned with the $y$-axis, the bias of the $y$-component is $\mathbf{- 2 . 0 4 \%}$. Relative mean standard deviations of the $x$ - and $y$-components are in the range $10 \%$ to $17 \%$ and below $3.2 \%$ for the $z$-component.
\end{abstract}

\section{INTRODUCTION}

3-D vector flow imaging (VFI) is needed to capture the full dynamics of the blood flow. Previously, several approaches to 3-D VFI have been presented [1]. These include methods using multiple probes [2]-[4], matrix probes [5]-[7], and row-column addressed probes [8]. Approaches using multiple probes are typically unable to cover full volumes at real-time frame rates and require accurate registration of the probes' relative positions.

Matrix probes allow the beam to be steered electronically in any direction [9]. In [5], a 1024 element matrix probe was used with a single plane wave emission limiting the insonified volume due to the small aperture. Holbek et al. [6] made line-by-line 3-D VFI in a single plane using the same probe, and volumetric VFI was not made. Wigen et al. [7] used a different probe with in-handle subaperture processing limiting the steering angle due to the appearance of grating lobes. Steered plane waves were emitted and the velocities were estimated within each insonified region from each steering direction. ECG-gating was used to combine velocity estimates from 5 heart-cycles into a single 3-D VFI volume or a thick slice. While row-column addressed probes show promising results [8], in-vivo 3-D VFI remains to be shown.

In this paper, a 3-D VFI method with a matrix array is presented based on transverse oscillations [10] combined with directional beamforming [11], synthetic aperture (SA) imaging [12], and a combination of emission sequence and velocity estimator that extends the upper limit on velocities that can be estimated [13]. SA techniques allow velocities to be estimated in the entire insonified volume on every emission removing the need for ECG-gating. Using defocused or focused waves with low F-numbers (as opposed to plane waves) expands the insonified volume to cover a pyramidical volume rather than a box-shaped volume.

Section II presents the methods and performance metrics used, Section III gives details of the simulations made, and Section IV presents the results. Section V discusses the results, and Section VI concludes the paper.

\section{Methods}

This section describes the acquisition sequence, the velocity estimator, and the performance metrics.

\section{A. Acquisition Sequence and Velocity Estimator}

The principles of the acquisition sequence and processing are illustrated in Fig. 1. A base synthetic aperture (SA) sequence is repeated indefinitely, and sequence instances $i$ and $i+1$ ( $i$ is odd) are interleaved such that emission 1 of sequence instance 1 is followed by emission 1 of sequence instance 2, which is then followed by emission 2 of sequence instance 1 and emission 2 of sequence instance 2, etc. For illustration purposes, Fig. 1 shows a sequence with only 2 emissions. Each emission results in a set of low-resolution lines called a low-resolution volume (LRV), and the LRVs from sequence instance $i$ are summed to form a high-resolution volume (HRV). The HRVs of sequence instances $i$ and $i+1(i$ odd) are correlated with each other to find the signal's spatial shift. A number of correlation functions are averaged to find a velocity estimate.

For each point in which the velocity should be estimated, three sets of lines are beamformed in each LRV, one set for each cardinal direction. For the $x$ - and $y$-directions, a set of directional lines are beamformed in the respective direction, centered around the estimation point, and spanning a pulse length axially. Two-peak Hanning apodizations are used to induce transverse oscillations [10] in the respective directions. The receive apodizations are shown in Fig. 2(a) for the $x$-direction and Fig. 2(b) for the $y$-direction. These lines are used in a directional transverse oscillation velocity estimator, where the axial oscillation is removed from the signal before correlation [13]. Each line in HRV $i$ is correlated with the corresponding line in HRV $i+1$ and the resulting correlation functions are averaged ( $i$ odd). This results in averaging across the pulse length. Additionally, the correlation 


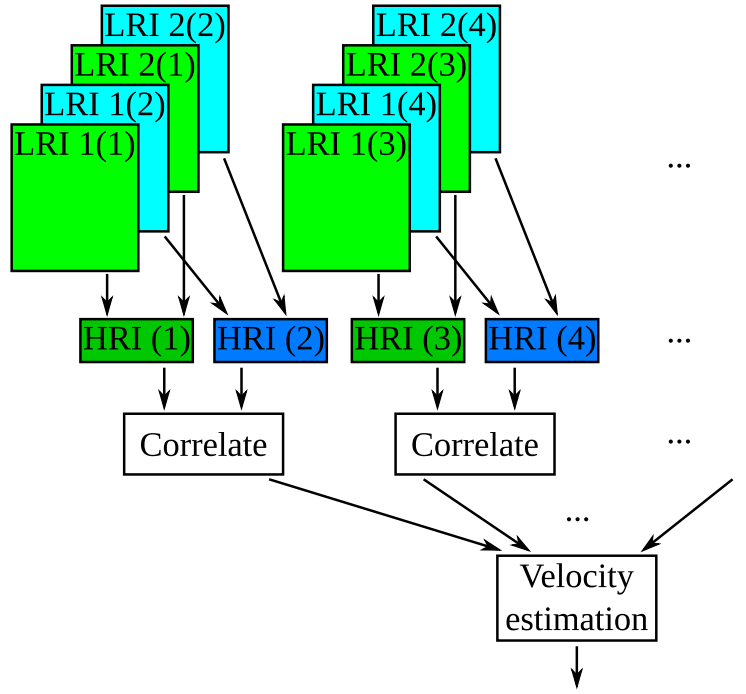

Fig. 1. Illustration of the acquisition sequence. The numbers in parentheses indicated the sequence instance number. Adapted from [13].

functions from a number of HRVs are averaged producing temporal averaging. The peak of the mean correlation function is found and parabolic interpolation is used to attain subsample precision. The resulting estimate of the signal's spatial shift is multiplied by $f_{p r f}$ to estimate the velocity component. For the $z$-direction, the lines are oriented along the $z$-axis, and the processing is the same, except for the removal of the axial oscillation.

The HRVs are separated in time by only $T_{p r f}=1 / f_{p r f}$, where $f_{\text {prf }}$ is the pulse repetition frequency. This raises the upper limit on velocities that can be estimated [13], [14] while allowing longer sequences to be used to reduce side-lobes for improved precision. Since the interleaved sequence is repeated indefinitely, continuous data is provided, which allows very low velocities to be estimated from the same data set.

\section{B. Performance Metrics}

The performance metrics consider the velocity components individually as well as the velocity magnitude. Averaging over a number, $N_{\text {avg }}$, of velocity estimates, the mean velocity in sample $s$ is $\bar{v}(s)$. The error to the true magnitude $v_{t}(s)$ is

$$
B(s)=\bar{v}(s)-v_{t}(s) .
$$

The performance measures are the component-wise relative mean velocity bias $\bar{B}_{v}$ and the relative mean velocity standard deviation $\overline{\sigma_{v}}$ as

$$
\bar{B}_{v}=\frac{1}{v_{0} N_{s}} \sum_{s=1}^{N_{s}} B(s)
$$

and

$$
\bar{\sigma}_{v}=\frac{1}{v_{0}} \sqrt{\frac{1}{N_{s}-1} \sum_{s=1}^{N_{s}} \sigma(\bar{v}(s))^{2}}
$$

where $v_{0}$ is the peak velocity in the vessel and $N_{s}$ is the number of discrete samples inside the vessel lumen.
TABLE I

Simulation AND Processing PaRAmeters

\begin{tabular}{l|l}
\multicolumn{2}{c}{ Transducer } \\
\hline \hline Number of columns & 35 \\
\hline Number of rows & 32 \\
\hline Missing column indices & 9,18, and 27 \\
\hline Pitch & $0.3 \mathrm{~mm}$ \\
\hline Center frequency, $f_{0}$ & $3 \mathrm{MHz}$ \\
\hline \multicolumn{2}{|c}{ Acquisition Sequence } \\
\hline \hline
\end{tabular}

\begin{tabular}{l|l}
\hline Number of emissions & 5
\end{tabular}

\begin{tabular}{l|l}
\hline Virtual source F-number & -2 \\
\hline
\end{tabular}

\begin{tabular}{ll}
\hline Virtual source steering angles & $0^{\circ}, \pm 6^{\circ}$ in $x, \pm 6^{\circ}$ in $y$ \\
\hline
\end{tabular}

\begin{tabular}{l|l}
\hline$f_{\text {prf }}$ & $15 \mathrm{kHz}$ \\
\hline
\end{tabular}

\begin{tabular}{l|l}
\hline Excitation pulse & 3 periods of a sinusoid at $f_{0}$ \\
\hline \hline
\end{tabular}

\begin{tabular}{ll|l}
\hline \hline Radius & $5 \mathrm{~mm}$ \\
\hline Depth & $30 \mathrm{~mm}$ \\
\hline Peak velocity & $0.5 \mathrm{~m} / \mathrm{s}$ \\
\hline \hline \multicolumn{2}{r}{} & \\
\hline
\end{tabular}

\begin{tabular}{l|l}
\hline \hline$x$ and $y$ & $0 \mathrm{~mm}$ \\
\hline$z$ & From $24.5 \mathrm{~mm}$ to $35.3 \mathrm{~mm}$ \\
\hline \hline \multicolumn{2}{c}{ Velocity Estimator }
\end{tabular}

\begin{tabular}{l|l}
\hline \hline Number of lines in pulse length & 24 \\
\hline
\end{tabular}

\begin{tabular}{l|l}
\hline Number of HRVs averaged & 16
\end{tabular}

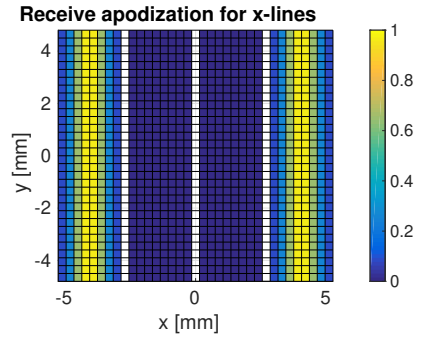

(a)

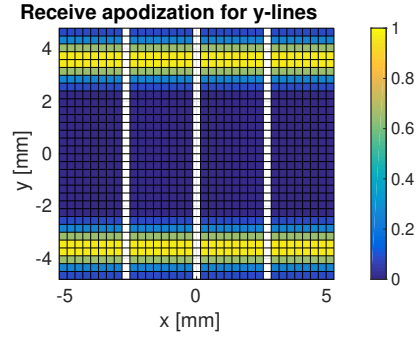

(b)
Fig. 2. The apodizations used in receive to create a transverse oscillation for the $x$-direction (a) and the $y$-direction (b).

\section{Simulations}

Field II Pro [15]-[17] simulations of a matrix array and a vessel with a parabolic flow profile were made. Two vessel orientations were simulated: parallel to the $x$-axis and parallel to the $y$-axis. The simulation parameters are given in Table I.

The simulated matrix array was the same as in [6], [18] and consisted of 4 blocks of $8 \times 32$ elements separated by a 1-element pitch. This is illustrated in Fig. 2 that also shows the two-peak apodizations used in receive.

800 emissions were simulated for each vessel orientation. Recursive SA imaging [19] was used to produce 396 pairs of 2 high-resolution images. Each pair was correlated with each other, and the correlation functions of 16 pairs were averaged to create one estimated velocity profile as described in Section II-A. A total of 24 estimated velocity profiles were produced, and the performance metrics were calculated across these. Performance metrics were calculated only on the estimates inside the vessel boundary. 
TABLE II

Relative Mean Velocity Bias and Standard DeViation IN PERCENT

\begin{tabular}{c||c|c||c|c}
\multicolumn{1}{c||}{} & \multicolumn{2}{c||}{$x$-direction } & \multicolumn{2}{c}{$y$-direction } \\
\hline & $\bar{B}$ & $\bar{\sigma}$ & $\bar{B}$ & $\bar{\sigma}$ \\
\hline \hline$v_{x}$ & -10.1 & 16.8 & 1.41 & 11.0 \\
\hline$v_{y}$ & -0.77 & 14.0 & -2.04 & 16.9 \\
\hline$v_{z}$ & 1.70 & 3.2 & -0.10 & 1.55 \\
\hline$|\mathbf{v}|$ & -4.2 & 16.7 & -1.53 & 16.6
\end{tabular}

\section{RESULTS}

Table II shows the relative mean velocity bias and standard deviation for both vessel orientations for all velocity components and the velocity magnitude. A bias of $-10.1 \%$ is found for the $v_{x}$ component with flow in the $x$-direction, while it is only $-2.04 \%$ for the $v_{y}$ component with flow in the $y$-direction. The relative mean standard deviations are higher for the $x$ - and $y$-directions $(10 \%$ to $17 \%)$ than for the $z$-direction $(\leq 3.2 \%)$. The bias of the velocity magnitude is $-4.2 \%$ for the vessel in the $x$-direction and $-1.53 \%$ for the vessel in the $y$-direction. Relative mean stadard deviations of the velocity magnitude are similar to those of the component in the flow direction.

Fig. 3 shows the estimated velocity components for the vessel in the $x$-direction on the left and the $y$-direction on the right. For the vessel aligned with the $x$-axis, a parabolic profile is seen for the $x$-component, and for the vessel aligned with the $y$-axis, a parabolic profile is seen for the $y$-component. For the vessel aligned with the $x$-axis, a small parabolic profile is seen as well for the $z$-component. All other velocity components are near-zero as expected.

\section{DISCUSSION}

The bias of the estimates in the flow direction are higher in the $x$-direction than in the $y$-direction $(-10.1 \%$ vs $-2.04 \%)$. At the same time, a small parabolic profile is seen in the $z$-component for flow in the $x$-direction, while all other components orthogonal to the flow are zero as expected. Going from the estimated velocity component in the flow direction to the velocity magnitude, the bias in the $x$-direction was reduced by $58 \%$ from $-10.1 \%$ to $-4.2 \%$, while the bias in the $y$ direction reduced $25 \%$ from $-2.04 \%$ to $-1.53 \%$. The gaps in the transducer array in the $x$-direction result in artefacts in the transmitted field, which give rise to grating lobes and cause deviations from the assumed spherical delay model in the beamforming. It is hypothesized that this may cause some of the signal to appear to move in the $z$-direction (the observed small parabolic profile), effectively changing the observed flow from purely lateral to having a small axial component. Further investigations are needed to confirm or reject this hypothesis.

\section{CONCLUSION}

A 3-D vector flow imaging method using a matrix transducer array and a directional transverse oscillations synthetic aperture velocity estimator was presented. Simulations show a relative mean bias of $-10.1 \%$ of the $x$-component with parabolic flow in the $x$-direction and $-2.04 \%$ of the $y$ component with parabolic flow in the $y$-direction. Relative mean standard deviations are in the range $10 \%$ to $17 \%$ for the $x$ - and $y$-components and $<3.2 \%$ for the $z$-component. This shows that 3-D VFI can be made with the proposed method.

\section{REFERENCES}

[1] J. A. Jensen, S. I. Nikolov, A. Yu, and D. Garcia, "Ultrasound vector flow imaging I: Sequential systems," IEEE Trans. Ultrason., Ferroelec., Freq. Contr., vol. 63, no. 11, pp. 1704-1721, 2016.

[2] M. D. Fox, "Multiple crossed-beam ultrasound Doppler velocimetry," IEEE Trans. Son. Ultrason., vol. SU-25, pp. 281-286, 1978.

[3] V. L. Newhouse, K. S. Dickerson, D. Cathignol, and J.-Y. Chapelon, "Three-dimensional vector flow estimation using two transducers and spectral width," IEEE Trans. Ultrason., Ferroelec., Freq. Contr., vol. 41, pp. 90-95, 1994.

[4] I. A. Hein, "Triple-beam lens transducers for three-dimensional ultrasonic fluid flow estimation," IEEE Trans. Ultrason., Ferroelec., Freq. Contr., vol. 42, pp. 854-869, 1995.

[5] M. J. Pihl and J. A. Jensen, "A transverse oscillation approach for estimation of three-dimensional velocity vectors. Part I: Concept and simulation study," IEEE Trans. Ultrason., Ferroelec., Freq. Contr., vol. 61, pp. 1599-1607, 2014.

[6] S. Holbek, C. Ewertsen, H. Bouzari, M. J. Pihl, K. L. Hansen, M. B Stuart, M. B. Nielsen, and J. A. Jensen, "Ultrasonic 3-D vector flow method for quantitative in vivo peak velocity and flow rate estimation," IEEE Trans. Ultrason., Ferroelec., Freq. Contr., vol. 64, no. 3, pp. 544 554, 2017.

[7] M. S. Wigen, S. Fadnes, A. Rodriguez-Molares, T. Bjåstad, M. Eriksen, K. H. Stensæth, A. Støylen, and L. Løvstakken, "4-D intracardiac ultrasound vector flow imaging-reasibility and comparison to phasecontrast MRI," IEEE Trans. Med. Imag., vol. 37, no. 12, pp. 2619-2629, December 2018.

[8] S. Holbek, T. L. Christiansen, M. B. Stuart, C. Beers, E. V. Thomsen, and J. A. Jensen, "3-D vector flow estimation with row-column addressed arrays," IEEE Trans. Ultrason., Ferroelec., Freq. Contr., vol. 63, no. 11, pp. 1799-1814, 2016.

[9] S. W. Smith, H. G. Pavy, and O. T. von Ramm, "High speed ultrasound volumetric imaging system - Part I: Transducer design and beam steering," IEEE Trans. Ultrason., Ferroelec., Freq. Contr., vol. 38, pp. 100-108, 1991.

[10] J. A. Jensen and P. Munk, "A new method for estimation of velocity vectors," IEEE Trans. Ultrason., Ferroelec., Freq. Contr., vol. 45, no. 3 , pp. 837-851, 1998.

[11] J. A. Jensen, "Directional velocity estimation using focusing along the flow direction: I: Theory and simulation," IEEE Trans. Ultrason., Ferroelec., Freq. Contr, vol. 50, pp. 857-872, 2003.

[12] S. I. Nikolov and J. A. Jensen, "In-vivo synthetic aperture flow imaging in medical ultrasound," IEEE Trans. Ultrason., Ferroelec., Freq. Contr., vol. 50, no. 7, pp. 848-856, 2003.

[13] J. A. Jensen, "Estimation of high velocities in synthetic aperture imaging: I: Theory," IEEE Trans. Ultrason., Ferroelec., Freq. Contr., vol. 66, no. 6, pp. 1024-1031, 2019.

[14] — - "Estimation of high velocities in synthetic aperture imaging: II: Experimental investigation," IEEE Trans. Ultrason., Ferroelec., Freq. Contr., vol. 66, no. 6, pp. 1032-1038, 2019.

[15] J. A. Jensen and N. B. Svendsen, "Calculation of pressure fields from arbitrarily shaped, apodized, and excited ultrasound transducers," IEEE Trans. Ultrason., Ferroelec., Freq. Contr., vol. 39, no. 2, pp. 262-267, 1992.

[16] J. A. Jensen, "Field: A program for simulating ultrasound systems," Med. Biol. Eng. Comp., vol. 10th Nordic-Baltic Conference on Biomedical Imaging, Vol. 4, Supplement 1, Part 1, pp. 351-353, 1996.

[17] — , "A multi-threaded version of Field II," in Proc. IEEE Ultrason. Symp. IEEE, 2014, pp. 2229-2232.

[18] M. J. Pihl, M. B. Stuart, B. G. Tomov, M. F. Rasmussen, and J. A. Jensen, "A transverse oscillation approach for estimation of threedimensional velocity vectors. Part II: Experimental validation," IEEE Trans. Ultrason., Ferroelec., Freq. Contr., vol. 51, no. 10, pp. 16081618, 2014.

[19] S. I. Nikolov, K. Gammelmark, and J. A. Jensen, "Recursive ultrasound imaging," in Proc. IEEE Ultrason. Symp., vol. 2, 1999, pp. 1621-1625. 


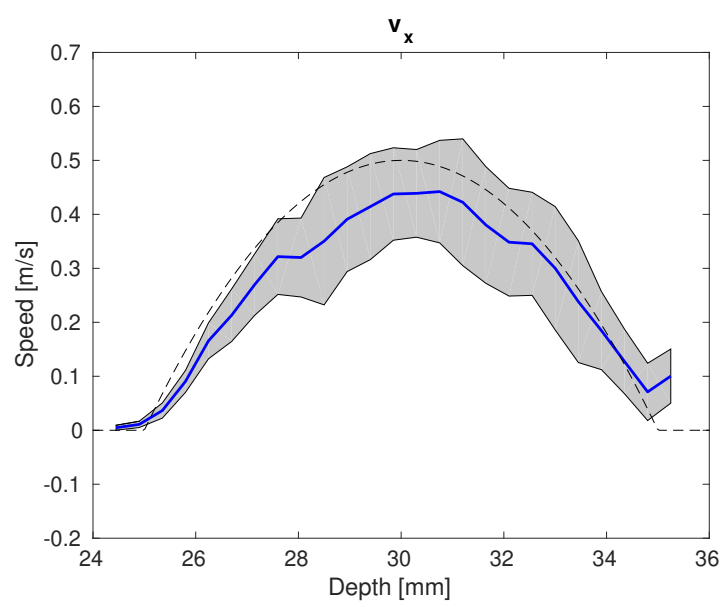

(a)

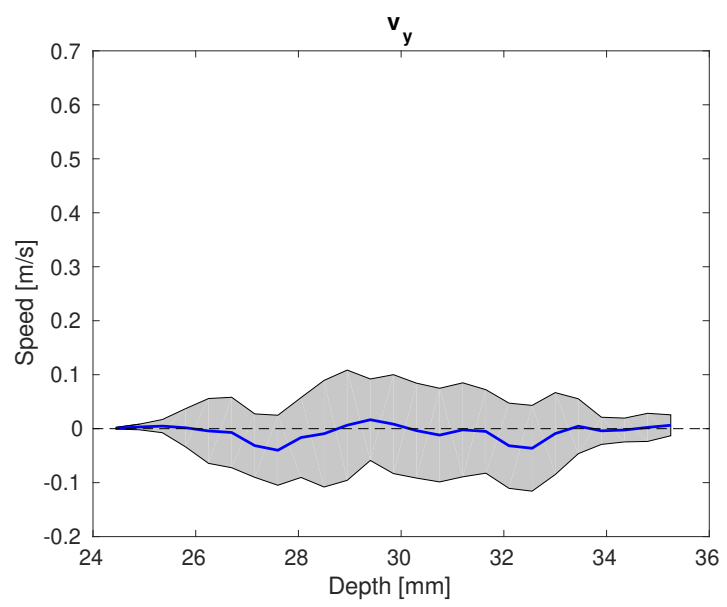

(c)

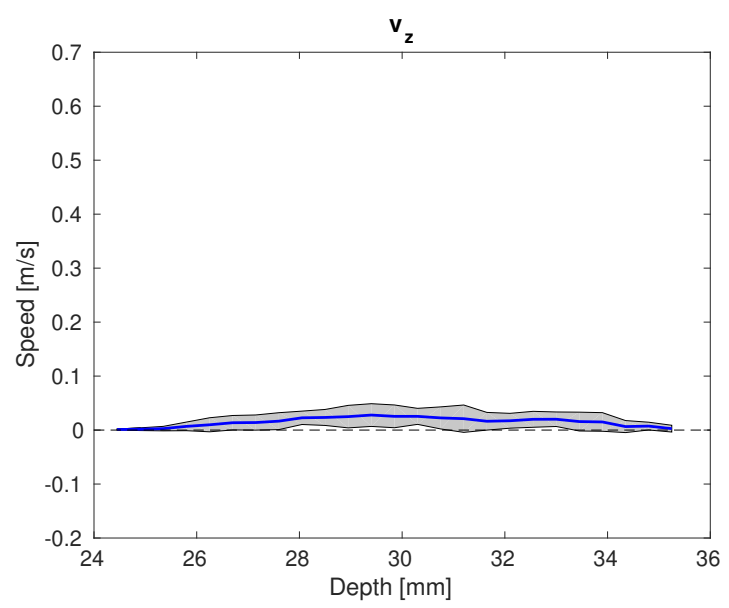

(e)

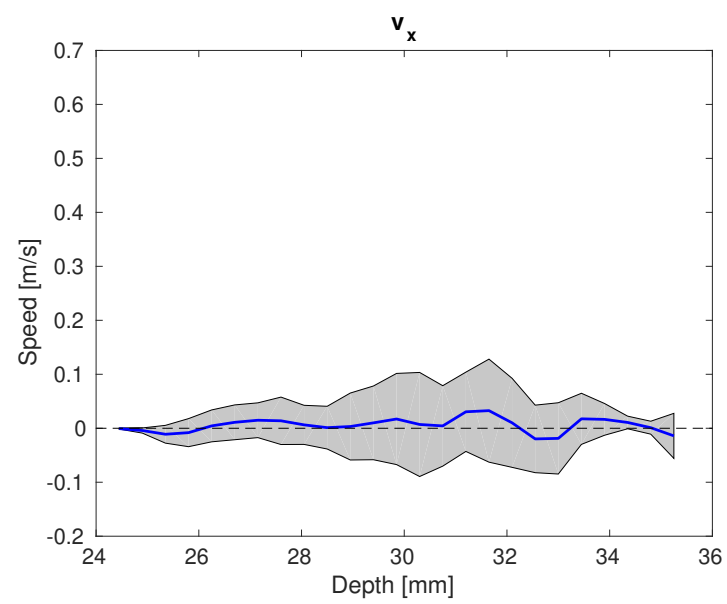

(b)

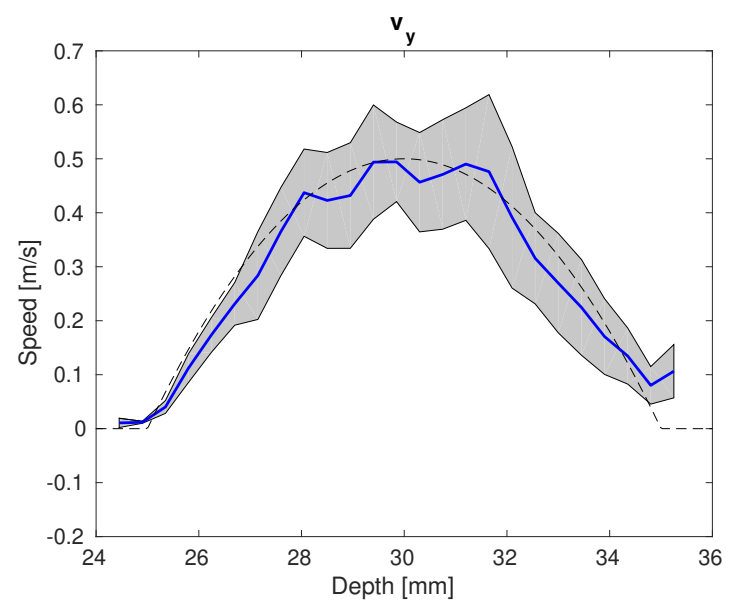

(d)

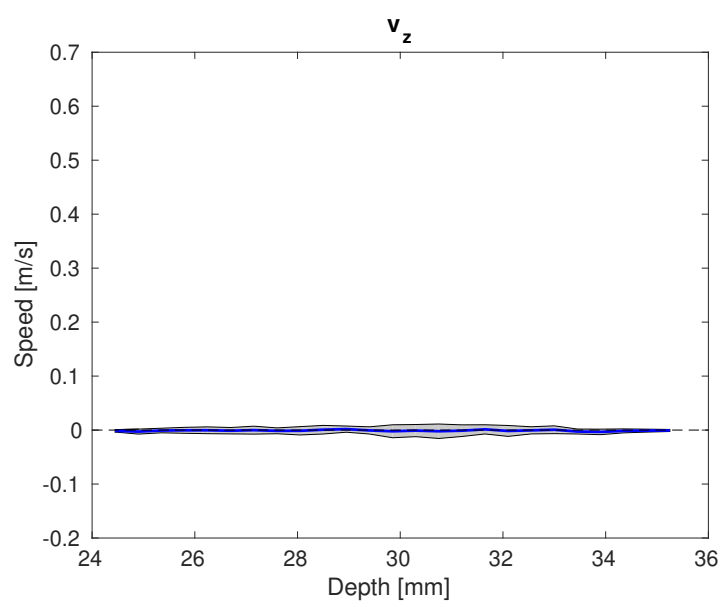

(f)

Fig. 3. Estimated velocity components for the vessel in the $x$-direction (a), (c), and (e) and the $y$-direction (b), (d), and (f). 